\title{
Deflection Routing in Complex Networks
}

\author{
Soroush Haeri and Ljiljana Trajković \\ Simon Fraser University \\ Vancouver, British Columbia, Canada \\ Email: \{shaeri, ljilja\}@sfu.ca
}

\begin{abstract}
Contention is the main source of information loss in buffer-less network architectures where deflection routing is a viable contention resolution scheme. In recent years, various reinforcement learning-based deflection routing algorithms have been proposed. However, performance of these algorithms has not been evaluated in larger networks that resemble the autonomous system-level topology of the Internet.
\end{abstract}

In this paper, we compare performance of three reinforcement learning-based deflection routing algorithms by using topologies generated with Waxman and Barabási-Albert algorithms. We examine the scalability of deflection routing algorithms by increasing the network size while keeping the network load constant.

\section{INTRODUCTION}

Deflection routing is a viable contention resolution scheme that may be employed in buffer-less networks such as networks on chips or optical burst-switched (OBS) networks. Contention occurs when according to a routing table, multiple arriving traffic flows at a node need to be routed through a single outgoing link. In this case, only one flow is routed through the optimal link defined by the routing table. In the absence of a contention resolution scheme, the remaining flows are discarded because the node possesses no buffers. Instead of buffering or discarding packets, deflection routing helps to temporarily deflect them away from the path that is prescribed by the routing table.

Optical burst switching [1] is a buffer-less architecture that has been introduced to eliminate the optical/electrical/optical signal conversions in high-performance optical networks. The optical/electrical conversion occurs when a data packet arrives at a router and the source and destination addresses required for routing should be extracted from the packet header. The electrical/optical conversion is then required to forward the packet through the appropriate outgoing link. High-speed optical links are often used to connect the Internet Autonomous Systems and, hence, the optical burst switching may be used for inter-autonomous system communications.

Insights emanating from the discovery of power-law distribution of nodes degree [2] and scale-free properties of communication networks [3], [4] have influenced the design of routing protocols [5], [6]. Various empirical results confirm the presence of power laws in the Internet's inter-autonomous system-level topologies [7]-[9]. Waxman [10] and BarabásiAlbert [3] algorithms have been widely used to generate Internet-like graphs.

Various deflection routing algorithms proposed in the literature have only been tested on small-size networks such as the National Science Foundation's network or general torus topologies that do not resemble the Internet [11]-[15].
In this paper, we compare performance of the Q-learningbased Node Degree Dependent (Q-NDD) [14] deflection routing algorithm, the Predictive Q-learning Deflection Routing (PQDR) [13] algorithm, and the Reinforcement Learningbased Deflection Routing Scheme (RLDRS) [12] by using randomly generated Waxman [10] and scale-free BarabásiAlbert [3] network topologies. The three deflection routing algorithms belong to the class of algorithms that rely on intelligence in a network node to deflect packets.

This paper is organized as follows. In Section II, we provide a brief survey of research related to deflection routing. We also describe the available algorithms for generating network topologies and briefly highlight their differences. Performance evaluation and simulation scenarios are presented in Section III. We conclude with Section IV.

\section{RELATED WORK}

\section{A. Deflection Routing}

Various deflection routing architectures have been proposed in the literature. Slotted and unslotted deflection schemes were compared [16], [17] and performance of a simple random deflection algorithm and loss rates of deflected data were analyzed [18]-[21]. Various integrations of deflection routing with wavelength conversion and fiber delay lines were also proposed [22], [23].

Deflection protocols were recently enhanced by enabling neighboring nodes to exchange traffic information. Hence, each node generates its deflection decisions based on better understanding of its surrounding [15], [24], [25]. Heuristic approaches may also be used to process the information gathered from the neighboring nodes.

Reinforcement learning techniques have been recently employed to generate deflection decisions. The Q-learning path selection algorithm [11] calculates a priori set of candidate paths $P=\left\{p_{1}, \ldots, p_{m}\right\}$ for tuples $\left(s_{i}, s_{j}\right)$, where $s_{i}, s_{j} \in S$ and $S=\left\{s_{1}, \ldots, s_{n}\right\}$ denotes the set of all edge nodes in the network. The $i^{t h}$ edge maintains a Q-table that contains a quality value (Q-value) for every tuple $\left(s_{j}, p_{k}\right)$, where $s_{j} \in S \backslash\left\{s_{i}\right\}$ and $p_{k} \in P$. The sets $S$ and $P$ are states and actions, respectively. The Q-value is updated after each decision is made and the score of the path is reduced or increased depending on the received rewards. The algorithm does not specify a signaling method or a procedure for handling feedback signals.

RLDRS [12] employs the Q-learning algorithm for deflection routing. The advantages of RLDRS are its precise signaling and rewarding procedures. Routing algorithms that are based on Q-learning do not generate optimal routing 
policies in networks with low loads nor do they learn new optimal policies in the cases when network load decreases.

The PQDR [13] algorithm employs the predictive Qrouting (PQR) [26] that addresses the shortcomings of $\mathrm{Q}$ learning-based routing by recording the best experiences learned, which may be reused to predict traffic behavior.

A drawback of the Q-learning path selection algorithm, RLDRS, and PQDR is their complexity, which depends on the size of the network. Hence, they are not easily scalable. The recently proposed Q-NDD algorithm [14] employs Q-learning for deflection routing. It scales well in larger networks because its complexity depends on the node degree rather than the network size. In the case of RLDRS and PQDR, nodes receive feedback signals for every packet that they deflect while in the case of Q-NDD, feedback signals are received only if the deflected packet is discarded by another node.

\section{B. Network Topologies}

Waxman [10] algorithm is commonly used to synthetically generate random network topologies. In a Waxman graph, an edge that connects nodes $u$ and $v$ exists with a probability:

$$
\operatorname{Pr}(\{u, v\})=\eta \exp \left(\frac{-d(u, v)}{L \delta}\right)
$$

where $d(u, v)$ is the distance between nodes $u$ and $v, L$ is the maximum distance between the two nodes, and $\eta$ and $\delta$ are parameters in the range $(0,1]$. Graphs generated with larger $\eta$ and smaller $\delta$ values contain larger number of short edges. These graphs have longer hop diameter, shorter length diameter, and larger number of bicomponents [27]. Graphs generated using Waxman algorithm do not resemble the backbone and hierarchal structure present in the Internet. Furthermore, the algorithm does not guarantee a connected network [28].

Barabási-Albert [3] algorithm is used to generate scale-free graphs that possess power-law distribution of node degrees. It suggests that incremental growth and preferential connectivity are possible causes for this power-law distribution. It has been established that Internet graphs on the Autonomous System level exhibit the power-law distribution properties of scale-free graphs [2], [8], [9]. Therefore, Barabási-Albert algorithm may be used to generate viable Internet-like graphs. The algorithm begins with a connected network of $n$ nodes. A new node $i$ that is added to the network will connects to an existing node $j$ with probability:

$$
\operatorname{Pr}(i, j)=\frac{d_{j}}{\sum_{k \in N} d_{k}},
$$

where $d_{j}$ denotes the degree of the node $j, N$ is the set of all nodes in the network, and $\sum_{k \in N} d_{k}$ is the sum of the degrees of all nodes in the network.

\section{Simulation Results}

In order to evaluate and compare performance of Q-NDD, PQDR, and RLDRS, we implement these algorithms in the ns3 network simulator [30]. We compare the algorithms in terms of burst-loss probability, number of deflections, and average number of hops.
We use the Boston University Representative Internet Topology Generator (BRITE) [29] to generate autonomous system-level topologies that consist of 10, 20, 50, 100, 200, 500 , and 1,000 nodes. These topologies are generated using the Waxman and Barabási-Albert algorithms.

We consider a buffer-less optical burst-switched architecture for data transmission where nodes are connected using bi-directional $1 \mathrm{Gbps}$ single wavelength fiber links. The nodes are randomly placed and each node is connected to three other nodes. Subsets of nodes are randomly selected as sources and destinations of Poisson traffic flows. Multiple Poisson flows having a data rate of $0.5 \mathrm{Gbps}$ are transmitted across the network. Each Poisson flow is 50 bursts long with each burst containing $12.5 \mathrm{kB}$ of payload. Each simulation scenario is repeated two times with various random assignments of nodes as sources and as destinations. Simulation results are averaged over two simulation runs. For each scenario, we keep the network load at 20\%. Hence, scenarios with 10, 20, 50, 100, 200, 500, and 1,000 nodes have 24, 48, 120, 240, 480, 1,200, and 2,400 Poisson traffic flows, respectively. Simulations were performed on a Dell Optiplex-790 with 16 GB memory and the Intel Core i7 2600 processor. Simulation scenarios are shown in Table I.

TABLE I. SUmmary OF Simulation Scenarios.

\begin{tabular}{c|c|c|c|c}
\hline $\begin{array}{c}\text { Topology } \\
\text { generating } \\
\text { algorithm }\end{array}$ & $\begin{array}{c}\text { Deflection } \\
\text { routing } \\
\text { algorithm }\end{array}$ & $\begin{array}{c}\text { Number } \\
\text { of } \\
\text { nodes }\end{array}$ & $\begin{array}{c}\text { Number } \\
\text { of } \\
\text { links }\end{array}$ & $\begin{array}{c}\text { Number } \\
\text { of } \\
\text { flows }\end{array}$ \\
\hline \multirow{4}{*}{$\begin{array}{c}\text { Waxman } \\
\text { Barabási-Albert }\end{array}$} & \multirow{2}{*}{$\begin{array}{c}\text { Q-NDD } \\
\text { PQDR }\end{array}$} & 10 & 30 & 24 \\
\cline { 3 - 5 } & RLDRS & 20 & 60 & 48 \\
\cline { 3 - 5 } & & 100 & 300 & 240 \\
\cline { 3 - 5 } & & 200 & 600 & 480 \\
\cline { 3 - 5 } & & 500 & 1,500 & 1,200 \\
\hline
\end{tabular}

\section{A. Burst-Loss Probability}

Performance of Q-NDD, PQDR, and RLDRS deflection routing algorithms in terms of burst-loss probability as a function of number of nodes for Waxman and Barabási-Albert network topologies is shown in Fig. 1. Burst-loss probability has a logarithmic trend. It is slightly higher in Barabási-Albert networks. Q-NDD scales better than PQDR and RLDRS as the size of the network grows.

\section{B. Number of Deflections}

Although burst deflection reduces the burst-loss probability, it introduces excess traffic load to the network. This behavior is undesired from the traffic engineering point of view. Therefore, the volume of the deflected traffic should also be considered as a performance measure. Number of deflections as a function of number of nodes for Waxman and Barabási-Albert network topologies is shown in Fig. 2. Q-NDD deflects fewer number of bursts compared to PQDR and RLDRS. Comparison of Waxman and Barabási-Albert network topologies shows an insignificant variation in the number of deflections. 

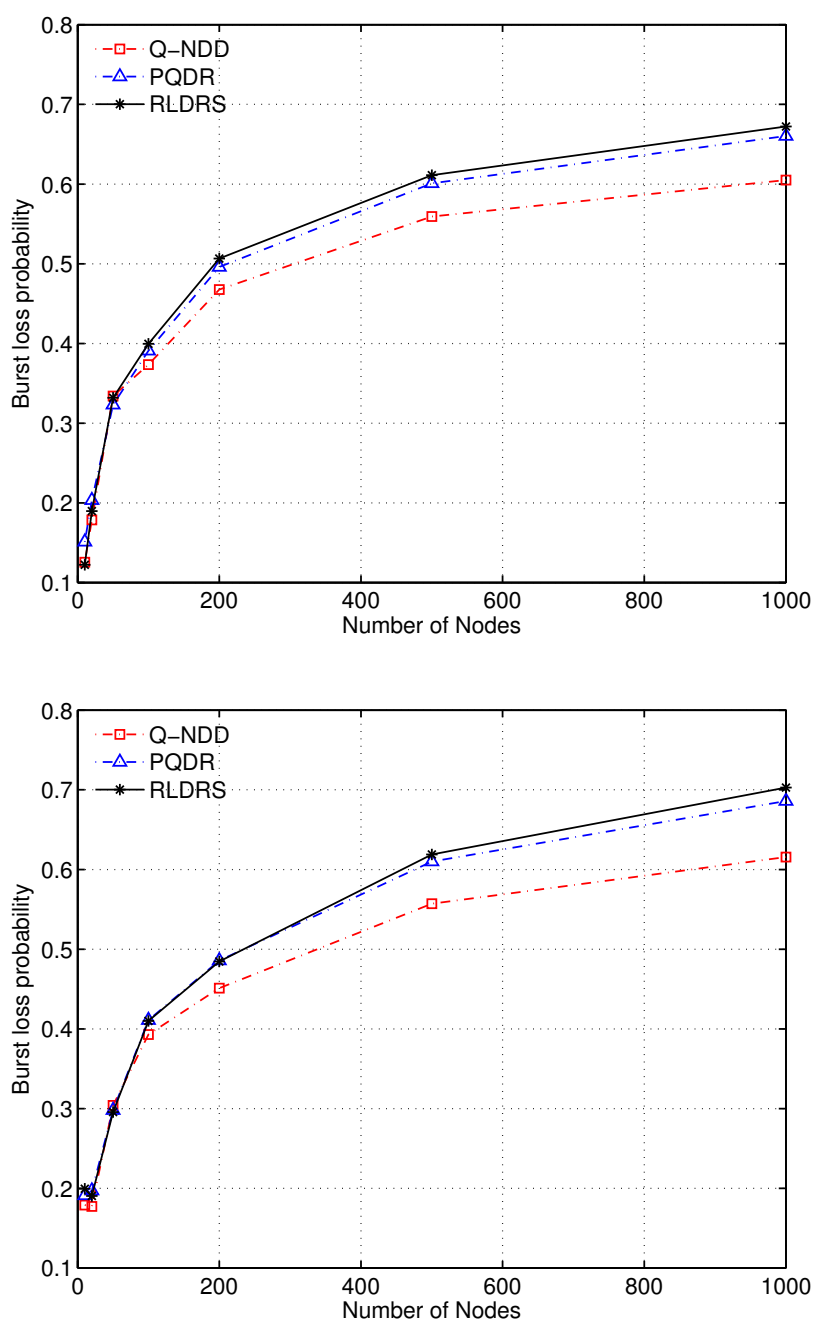

Fig. 1. Burst loss probability as a function of the number of nodes in Waxman (top) and Barabási-Albert (bottom) networks.

\section{Average Number of Hops}

Average number of hops traveled by bursts as a function of the number of network nodes for Waxman and Barabási-Albert topologies is shown in Fig. 3. In case of Q-NDD, bursts travel through more hops compared to PQDR and RLDRS. When deflecting a burst, PQDR and RLDRS consider the number of hops to destination. Simulation results show that the underlying topology and nodes connectivity have an impact on the number of hops traveled by bursts. Bursts travel fewer hops in case of Barabási-Albert networks. Consider the case of 1,000 nodes shown in Fig. 3. In the case of Waxman topology shown in Fig. 3 (top), the Q-NDD algorithm causes bursts to travel 3.5 hops on average while in the case of Barabási-Albert topology shown in Fig. 3 (bottom), number of traveled hops is only 2.75 .

\section{CONCLUSiON}

In this paper, we compared performance of the Q-learningbased Node Degree Dependent (Q-NDD) deflection routing algorithm, the Predictive Q-learning-based Deflection Routing
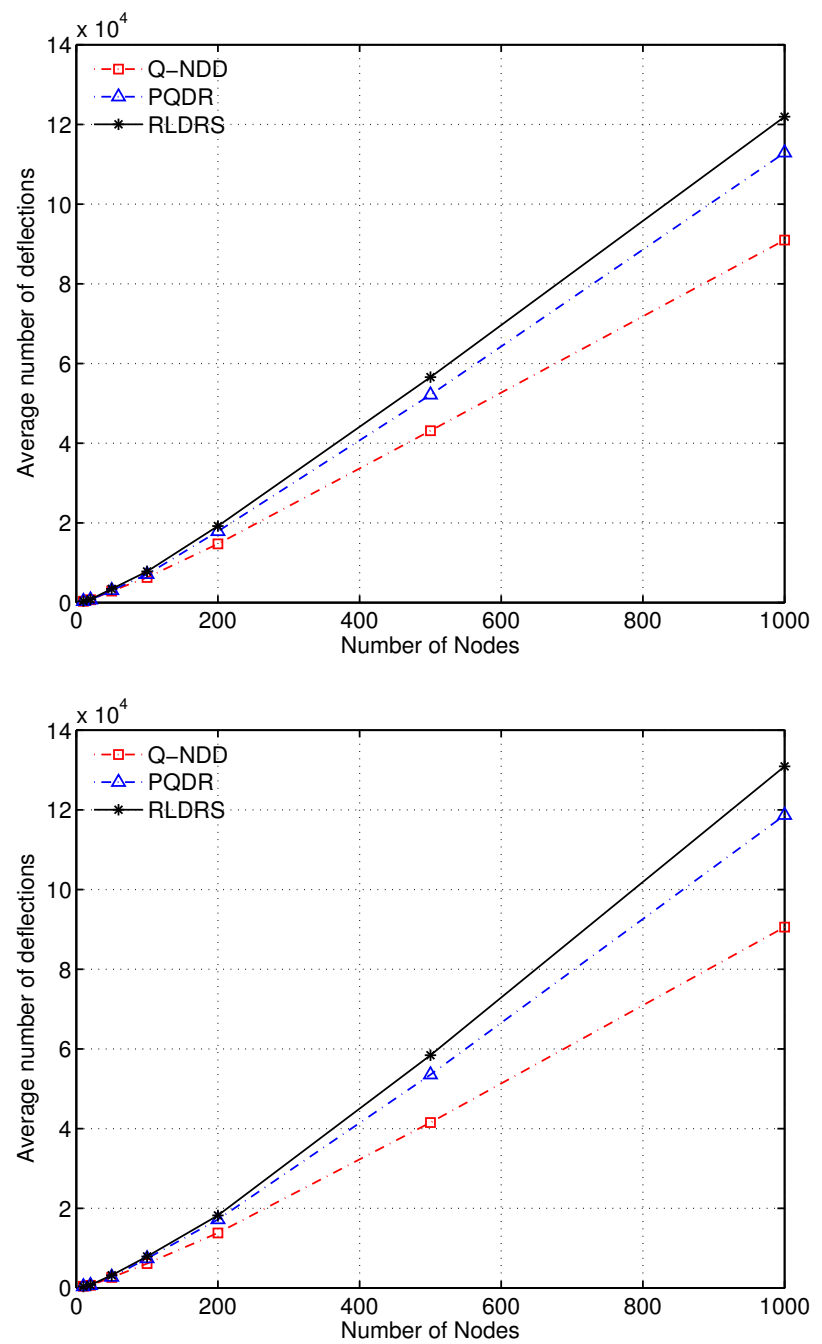

Fig. 2. Number of deflections as a function of the number of nodes in Waxman (top) and Barabási-Albert (bottom) networks.

(PQDR) algorithm, and the Reinforcement Learning Based Deflection Routing Scheme (RLDRS). Simulations were performed using complex network topologies that were generated by Waxman and Barabási-Albert algorithms.

The Q-NDD burst-loss probability is smaller and bursts are deflected less frequently. However, bursts travel through more hops and thus experience longer end-to-end delays. Therefore, smaller burst-loss probability and smaller number of deflections come at the cost of selecting longer paths, which are less likely to be congested. PQDR and RLDRS consider the number of hops to destination when deflecting bursts. This, in turn, causes the bursts to travel through shorter paths. However, the probability of congestion along shorter paths is usually higher because the majority of the routing protocols tend to route data through such paths. As a result, burst-loss probability and probability of defecting bursts is higher along the paths that PQDR and RLDRS select for deflection.

While the simulation results indicate that number of deflections does not significantly depend on the underlying topology, the bursts travel through fewer hops in Barabási-Albert net- 

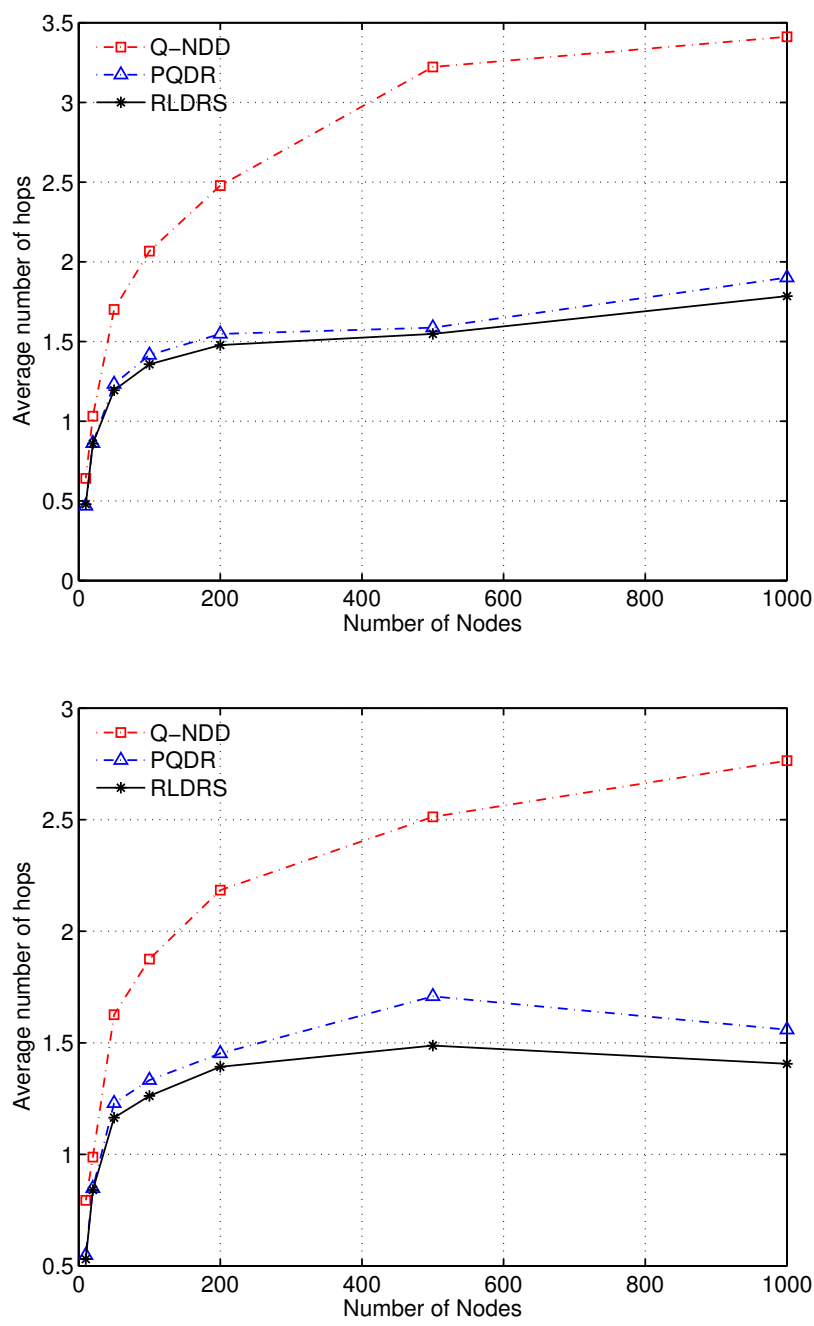

Fig. 3. Average number of hops traveled by bursts as a function of the number of nodes in Waxman (top) and Barabási-Albert (bottom) networks.

works. Q-NDD outperforms PQDR and RLDRS by exhibiting smaller burst-loss probability and number of deflections. However, in the case of Q-NDD, the bursts travel through additional hops. The improved performance of Q-NDD becomes more evident as the size of the network increases.

\section{REFERENCES}

[1] C. Qiao and M. Yoo, "Optical burst switching (OBS) - a new paradigm for an optical Internet," J. High Speed Netw., vol. 8, no. 1, pp. 69-84, Mar. 1999.

[2] M. Faloutsos, P. Faloutsos, and C. Faloutsos, "On power-law relationships of the Internet topology," SIGCOMM Comput. Commun. Rev., vol. 29, no. 4, pp. 251-262, Aug. 1999.

[3] A.-L. Barabási and R. Albert, "Emergence of scaling in random networks," Science, vol. 286, no. 5439, pp. 509-512, Oct. 1999.

[4] R. Albert and A.-L. Barabási, "Statistical mechanics of complex networks," Rev. Mod. Phys., vol. 74, pp. 47-97, Jan. 2002.

[5] W.-X. Wang, C.-Y. Yin, G. Yan, and B.-H. Wang, "Integrating local static and dynamic information for routing traffic," Phys. Rev. E, vol. 74, p. 016101, July 2006.

[6] J. Lü, G. Chen, M. Ogorzalek, and Lj. Trajković, "Theories and applications of complex networks: advances and challenges," in Proc. IEEE Int. Symp. Circuits and Systems, Beijing, China, May 2013, pp. 2291-2294.
[7] Lj. Trajković, "Analysis of Internet topologies," IEEE Circuits and Systems Mag., vol. 10, no. 3, pp. 48-54, Third Quarter 2010.

[8] L. Subedi and Lj. Trajković, "Spectral analysis of Internet topology graphs," in Proc. IEEE Int. Symp. Circuits and Systems, Paris, France, June 2010, pp. 1803-1806.

[9] M. Najiminaini, L. Subedi, and Lj. Trajković, "Analysis of Internet topologies: a historical view," in Proc. IEEE Int. Symp. Circuits and Systems, Taipei, Taiwan, May 2009, pp. 1697-1700.

[10] B. M. Waxman, "Routing of multipoint connections," IEEE J. Sel. Areas Commun., vol. 6, no. 9, pp. 1617-1622, Dec. 1988.

[11] Y. Kiran, T. Venkatesh, and C. Murthy, "A reinforcement learning framework for path selection and wavelength selection in optical burst switched networks," IEEE J. Sel. Areas Commun., vol. 25, no. 9, pp. 1826, Dec. 2007.

[12] A. Belbekkouche, A. Hafid, and M. Gendreau, "Novel reinforcement learning-based approaches to reduce loss probability in buffer-less OBS networks," Comput. Netw., vol. 53, no. 12, pp. 2091-2105, Aug. 2009.

[13] S. Haeri, M. Arianezhad, and Lj. Trajković, "A predictive Q-learningbased algorithm for deflection routing in buffer-less networks," in IEEE Int. Conf. Systems, Man, and Cybernetics (SMC 2013), Manchester, UK, Oct. 2013, pp. 764-769.

[14] S. Haeri, W. W-K. Thong, G. Chen, and Lj. Trajković, "A reinforcement learning-based algorithm for deflection routing in optical burst-switched networks," in IEEE Int. Conf. Inf. Reuse and Integration, San Francisco, USA, Aug. 2013, pp. 474-481.

[15] W. W.-K. Thong, G. Chen, and Lj. Trajković, "RED-f routing protocol for complex networks," in Proc. IEEE Int. Symp. Circuits and Systems, Seoul, Korea, May 2012, pp. 1644-1647.

[16] F. Borgonovo, L. Fratta, and J. Bannister, "Unslotted deflection routing in all-optical networks," in Proc. IEEE GLOBECOM, Houston, TX, Dec. 1993, pp. 119-125.

[17] T. Chich, J. Cohen, and P. Fraigniaud, "Unslotted deflection routing: a practical and efficient protocol for multihop optical networks," IEEE/ACM Trans. Netw., vol. 9, no. 1, pp. 47-59, Feb. 2001.

[18] F. Borgonovo, "Deflection routing," in Routing in Communications Networks. New Jersey: Prentice-Hall., 1995, pp. 263-306.

[19] A. Greenberg and B. Hajek, "Deflection routing in hypercube networks," IEEE Trans. Commun., vol. 40, no. 6, pp. 1070-1081, June 1992.

[20] A. Zalesky, H. Vu, Z. Rosberg, E. W. M. Wong, and M. Zukerman, "Stabilizing deflection routing in optical burst switched networks," IEEE J. Sel. Areas Commun., vol. 25, no. 6, pp. 3-19, Aug. 2007.

[21] E. W. M. Wong, J. Baliga, M. Zukerman, A. Zalesky, and G. Raskutti, "A new method for blocking probability evaluation in OBS/OPS networks with deflection routing," J. Lightw. Technol., vol. 27, no. 23, pp. 53355347, Dec. 2009.

[22] C. Q. Myungsik Yoo and S. Dixit, "Comparative study of contention resolution policies in optical burst-switched WDM networks," in Proc. SPIE, Boston, MA, Oct. 2000, vol. 4213, pp. 124-135.

[23] A. Zalesky, H. Vu, Z. Rosberg, E. Wong, and M. Zukerman, "OBS contention resolution performance," Performance Evaluation, vol. 64, no. 4, pp. 357-373, May 2007.

[24] X. Gao and M. Bassiouni, "Improving fairness with novel adaptive routing in optical burst-switched networks," J. Lightw. Technol., vol. 27, no. 20, pp. 4480-4492, Oct. 2009.

[25] J. Perelló, F. Agraz, S. Spadaro, J. Comellas, and G. Junyent, "Using updated neighbor state information for efficient contention avoidance in OBS networks," Comput. Commun., vol. 33, no. 1, pp. 65-72, Jan. 2010.

[26] S. P. M. Choi and D. -Y. Yeung, "Predictive Q-routing: a memory-based reinforcement learning approach to adaptive traffic control," in Advances in Neural Inform. Process. Syst., vol. 8, pp. 945-951, 1996.

[27] E. W. Zegura, K. L. Calvert, and M. J. Donahoo, "A quantitative comparison of graph-based models for Internet topology," IEEE/ACM Trans. Netw., vol. 5, no. 6, pp. 770-783, Dec. 1997.

[28] K. L. Calvert, M. B. Dora, and E. W. Zegura, "Modeling Internet topology," IEEE Commun. Mag., vol. 35, no. 6, pp. 160-163, June 1997.

[29] (2014, Feb. 14) BRITE. [Online]. Available: http://www.cs.bu.edu/brite.

[30] (2014, Feb. 14) The ns-3 network simulator. [Online]. Available: http://www.nsnam.org/. 